\title{
HOMOLOGICAL REALIZATION OF PRESCRIBED ABELIAN GROUPS VIA $K$-THEORY
}

\author{
A. J. BERRICK AND M. MATTHEY
}

\begin{abstract}
Using algebraic and topological $K$-theory together with complex $C^{*}$-algebras, we prove that every abelian group may be realized as the centre of a strongly torsion generated group whose integral homology is zero in dimension one and isomorphic to two arbitrarily prescribed abelian groups in dimensions two and three.
\end{abstract}

\section{INTRODUCTION AND STATEMENT OF THE MAIN RESULTS}

The main theorem of this paper combines two genres that we now describe.

(1) The first is the "inverse realization problem" for functors taking grouptheoretic values. The oldest example, still open, asks which finite groups can occur as Galois groups of rational polynomials [27. Another example is the theorem that every abelian group can be the ideal class group of a Dedekind domain 11, 24 . Eilenberg and Mac Lane solved the problem for the homotopy group functors 12 . For homology (always integral in this note), Baumslag, Dyer and Miller showed that every sequence of abelian groups could be realized as the reduced homology of a (discrete) group 3]. When one requires the group to be rich in torsion, the matter becomes more delicate. For finite groups, for instance, there are well-known constraints due to Maschke (cohomological version quoted in [19, p. 227]), Evens [13], and Swan [38. In the same vein, recall Milgram's counterexample in [28] to the conjecture (attributed to Loday) that no nontrivial finite group can have its first three positive-dimensional homology groups zero, see 16. Since any group with a series of finite length whose factors are either infinite cyclic or locally finite has the direct sum of all its reduced homology groups either infinite or zero 9], it is apparent that one needs to focus on a more general class of groups with torsion.

Progress has been made with the class of torsion-generated groups, wherein every element is a product of elements of finite order. In this case, there is a vestigial version of the previous result: if the sequence of homology groups of a torsiongenerated group is finite, then the group itself is perfect (that is, the first homology vanishes) 10. After a partial result in 7, exploiting the results on the ideal class group referred to above, the problem was settled by [10, as follows:

Let $A_{2}, A_{3}, \ldots$ be a sequence of abelian groups. Then there exists a strongly torsion generated group $G$ such that $H_{n}(G) \cong A_{n}$ for all $n \geq 2$.

Date: 28 March, 2004.

1991 Mathematics Subject Classification. Primary 19D55, 19K99, 20F99; Secondary 19C09, 20K40, 55N99.

Key words and phrases. Strongly torsion generated groups, homology, $K$-theory, $C^{*}$-algebras.

Research partially supported by NUS Research Grant R-146-000-049-112 for both authors, and partially by Swiss National Science Foundation for the second author. 
A strongly torsion generated group $G$ is one with the property that, for each $n \geq 2$, there is an element $g_{n}$ of order $n$ that normally generates $G$, in other words, every element of $G$ is a product of conjugates of $g_{n}$. The constraint $n \geq 2$ in the above statement occurs because such groups are necessarily perfect [10, Lem. 7]. Various properties of the class of strongly torsion generated groups are discussed in 10. It was introduced in 7 because its most notable examples arise in connection with algebraic $K$-theory. They include the infinite alternating group $A_{\infty}$ and the infinite special linear groups $\operatorname{SL}(\mathbb{Z})$ and $\operatorname{SL}(K)$ for any field $K$. The proof of the above theorem combines techniques of combinatorial group theory with Miller's affirmation, in [29], of the Sullivan Conjecture in homotopy theory.

(2) The second class of results that forms background to the present work consists of the embedding theorems in combinatorial group theory. For half a century it has been known that every group embeds in an algebraically closed group; these groups are strongly torsion generated [31, 35. When the embedded group is abelian, it is natural to attempt to embed it as the centre of the larger group. An embedding as the centre of a strongly torsion generated group was achieved in [7, again by means of an algebraic $K$-theory use of the result mentioned earlier on the ideal class group, and in [10] by means of combinatorial group theory and homotopy theory. (See also [17, 32 for constructions when the abelian group is locally finite.)

Whereas past displays of abelian groups as homology groups have formed separate results from realizations as centres, here we are able to combine these two strands in a single realization theorem, as follows.

Theorem 1.1. Let $A, B$ and $C$ be three abelian groups. Then, there exists a group $S$ with the following properties:

(i) $S$ is strongly torsion generated;

(ii) the centre of $S$ is isomorphic to $A$, that is, $\mathcal{Z}(S) \cong A$;

(iii) $S$ is perfect, that is, $H_{1}(S)=0$;

(iv) the second homology of $S$ is isomorphic to $B$, that is, $H_{2}(S) \cong B$;

(v) the third homology of $S$ is isomorphic to $C$, that is, $H_{3}(S) \cong C$.

The construction of $S$ is presented in Section 4 followed in Section 5 by the proof of the theorem. In Section [6 we collect further information on $S$ as a second theorem. The approach is based on a specific idea of [7] - related to the functors $K_{1}^{\text {alg }}$ and $K_{2}^{\text {alg }}$ for rings - and on some known results on both the topological and algebraic $K$-theory of complex $C^{*}$-algebras; this is all recalled in Sections 2 and 3. Section 7 poses some open questions on the subject, including two that are planned as the basis for further studies.

Acknowledgements : The second named author wishes to express his deep gratitude to Paul Balmer for fruitful discussions.

\section{Recollection on topological $K$-TheOry of $C^{*}$-ALGEBRAS}

The present section is devoted to some preparatory material on topological $K$ theory, needed for the proofs of our main results.

In the present paper, by a $C^{*}$-algebra, we always mean a complex $C^{*}$-algebra. Recall that the topological $K$-theory of $C^{*}$-algebras has the following properties: it is additive, that is, $K_{*}^{\text {top }}\left(\mathcal{A}_{1} \times \mathcal{A}_{2}\right) \cong K_{*}^{\text {top }}\left(\mathcal{A}_{1}\right) \oplus K_{*}^{\text {top }}\left(\mathcal{A}_{2}\right)$; it satisfies Bott periodicity, i.e. $K_{*}^{\text {top }}(\mathcal{A}) \cong K_{*+2}^{\text {top }}(\mathcal{A})$; it is continuous, namely, it commutes with 
filtered colimits (direct limits of $C^{*}$-algebras); and it is Morita invariant in the sense that there is an isomorphism $K_{*}^{\text {top }}\left(M_{n}(\mathcal{A})\right) \cong K_{*}^{\text {top }}(\mathcal{A})$. Let $\mathcal{K} \cong \operatorname{colim}_{n} M_{n}(\mathbb{C})$ be the $C^{*}$-algebra of compact operators on a separable complex Hilbert space, and $\widehat{\otimes}$ the minimal (i.e. spatial) tensor product of $C^{*}$-algebras. Since $M_{n}(\mathcal{A}) \cong \mathcal{A} \widehat{\otimes} M_{n}(\mathbb{C})$, combining Morita invariance and continuity, we deduce that topological $K$-theory is stable, in the sense that there is an isomorphism $K_{*}^{\text {top }}(\mathcal{A} \widehat{\otimes} \mathcal{K}) \cong K_{*}^{\text {top }}(\mathcal{A})$. The additivity, Bott and stability isomorphisms are canonical and natural. For $n \in \mathbb{Z}$, we also recall that

$$
K_{n}^{\mathrm{top}}(\mathbb{C}) \cong\left\{\begin{array} { l } 
{ \mathbb { Z } , \text { if } n \text { is even } } \\
{ 0 , \text { if } n \text { is odd } }
\end{array} \text { so that } \quad K _ { n } ^ { \mathrm { top } } ( \mathcal { K } ) \cong \left\{\begin{array}{l}
\mathbb{Z}, \text { if } n \text { is even } \\
0, \text { if } n \text { is odd }
\end{array}\right.\right.
$$

For more details on $C^{*}$-algebras and their topological $K$-theory (in particular for the properties we have recalled), we refer, for instance, to the books [33] and 41].

We need the following standard result from the theory of $C^{*}$-algebras and their topological $K$-theory. For a proof, we refer to [41, Ex. 9.H, pp. 173-174], where it is attributed to Higson and Brown; see also 33. for $M$ countable.

Proposition 2.1. For any abelian group $M$, there exists a $C^{*}$-algebra $\mathcal{E}_{M}$, whose topological $K$-theory is given by

$$
K_{2 n}^{\mathrm{top}}\left(\mathcal{E}_{M}\right) \cong M \quad \text { and } \quad K_{2 n+1}^{\mathrm{top}}\left(\mathcal{E}_{M}\right)=0 \quad(n \in \mathbb{Z}) .
$$

Remark 2.2. By construction, the $C^{*}$-algebra $\mathcal{E}_{M}$ of Proposition 2.1 is not unital.

Remark 2.3. The proof we give below of Theorem 1.1 is presented in such a way that if there is a construction of the $C^{*}$-algebra $\mathcal{E}_{M}$ in Proposition 2.1 that is functorial in $M$, then the group $S$ in Theorem[1.1] is also functorial in the abelian groups $A, B$ and $C$ on which it depends, and the homomorphisms occurring in its statement are all natural. Such a construction of $\mathcal{E}_{M}$ would certainly be of independent interest.

\section{Recollection on ALgebraid $K$-theory}

We now proceed with a recollection of standard - though sometimes highly nontrivial - results on algebraic $K$-theory needed in the proof of Theorem 1.1 that is presented in Section 5 below. As general references for algebraic $K$-theory, we mention the books 4], 26] and 34.

Let $\Lambda$ be a unital ring. Denote by $\mathrm{GL}(\Lambda)=\bigcup_{n \geq 1} \mathrm{GL}_{n}(\Lambda), \mathrm{E}(\Lambda)=\bigcup_{n \geq 1} \mathrm{E}_{n}(\Lambda)$ and $\operatorname{St}(\Lambda)=\operatorname{colim}_{n \geq 3} \operatorname{St}_{n}(\Lambda)$ the group of infinite invertible matrices, the group of infinite elementary matrices, and the infinite Steinberg group respectively. By definition, we have $K_{1}^{\mathrm{alg}}(\Lambda):=\mathrm{GL}(\Lambda) / \mathrm{E}(\Lambda)$, and, by the Whitehead Lemma, the equalities $[\mathrm{GL}(\Lambda), \mathrm{GL}(\Lambda)]=[\mathrm{E}(\Lambda), \mathrm{E}(\Lambda)]=\mathrm{E}(\Lambda)$ hold (see Milnor 30, Lem. 3.1]); in particular, the group $\mathrm{E}(\Lambda)$ is perfect and $K_{1}^{\text {alg }}(\Lambda)=H_{1}(\operatorname{GL}(\Lambda))$. By definition of $K_{2}^{\text {alg }}$ (cf. [30, p. 40]), we have a functorial exact sequence

$$
0 \longrightarrow K_{2}^{\mathrm{alg}}(\Lambda) \longrightarrow \mathrm{St}(\Lambda) \stackrel{\varphi_{\Lambda}}{\longrightarrow} \mathrm{GL}(\Lambda) \longrightarrow K_{1}^{\mathrm{alg}}(\Lambda) \longrightarrow 0
$$

with $\operatorname{Im}\left(\varphi_{\Lambda}\right)=\mathrm{E}(\Lambda)$. There are isomorphisms $K_{2}^{\text {alg }}(\Lambda) \cong \mathcal{Z}(\operatorname{St}(\Lambda)) \cong H_{2}(\mathrm{E}(\Lambda))$, and $\operatorname{St}(\Lambda)$ has vanishing $H_{1}$ and $H_{2}$, and is the universal central extension of $\mathrm{E}(\Lambda)$, see [30, Thms. 5.1 and 5.10]. For a perfect group $P$, one has $\mathcal{Z}(P / \mathcal{Z}(P))=0$ (see for instance [8] end of $\S 2.2])$. Therefore, $\mathrm{E}(\Lambda) \cong \operatorname{St}(\Lambda) / \mathcal{Z}(\operatorname{St}(\Lambda))$ is centreless. 
It is well-known that $K_{n}^{\mathrm{alg}}(\Lambda)$ is isomorphic to $\pi_{n}\left(B \mathrm{GL}(\Lambda)^{+}\right.$) for $n \geq 1$ (this is even the definition for $n \geq 3)$, to $\pi_{n}\left(B \mathrm{E}(\Lambda)^{+}\right)$for $n \geq 2$, and to $\pi_{n}\left(B \operatorname{St}(\Lambda)^{+}\right)$for $n \geq 3$ (see [34, Cor. 5.2.8]). Recall from [34, Thm. 5.2.2] that $H_{*}(X) \cong H_{*}\left(X^{+}\right)$ holds for any connected CW-complex $X$, as for example $B \mathrm{GL}(\Lambda), B \mathrm{E}(\Lambda)$ and $B \operatorname{St}(\Lambda)$. In particular, knowing that $B \mathrm{E}(\Lambda)^{+}$is 1-connected and that $B \operatorname{St}(\Lambda)^{+}$is 2-connected (cf. [34] Thm. 5.2.2]), by the Hurewicz Theorem [39] Thm. 10.25], the Hurewicz homomorphism induces the following epimorphisms and isomorphism:

$$
K_{3}^{\mathrm{alg}}(\Lambda) \rightarrow H_{3}(\mathrm{E}(\Lambda)), \quad K_{3}^{\mathrm{alg}}(\Lambda) \cong H_{3}(\operatorname{St}(\Lambda)) \quad \text { and } \quad K_{4}^{\mathrm{alg}}(\Lambda) \rightarrow H_{4}(\operatorname{St}(\Lambda))
$$

(the isomorphism is Gersten's Theorem [14]). All indicated isomorphisms and epimorphisms are canonical and natural. From [7, Lem. 1 and proof of Thm. A], we also quote that:

For a unital ring $\Lambda$, the groups $\mathrm{E}(\Lambda)$ and $\operatorname{St}(\Lambda)$ are strongly torsion generated.

For the definition of negative $K$-theory of a unital ring $\Lambda, K_{-n}^{\text {alg }}(\Lambda)$ with $n>0$, we refer to [34, Def. 3.3.1]. If $I$ is a nonunital ring, following [34 Def. 1.5.6], we define the minimal unitalization $\widetilde{I}$ of $I$ as the unital ring given, as a $\mathbb{Z}$-module, by the direct sum $\widetilde{I}:=I \oplus \mathbb{Z}$, and equipped with the multiplication given by

$$
(x, \lambda) \cdot\left(x^{\prime}, \lambda^{\prime}\right):=\left(x x^{\prime}+\lambda x^{\prime}+\lambda^{\prime} x, \lambda \lambda^{\prime}\right), \quad \text { for } x, x^{\prime} \in I \text { and } \lambda, \lambda^{\prime} \in \mathbb{Z} .
$$

As in [34. Def. 1.5.7], there is a split short exact sequence of nonunital rings

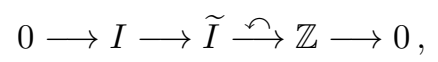

and one defines $K_{*}^{\text {alg }}(I)$ as to be the kernel of the map $K_{*}^{\text {alg }}(\widetilde{I}) \longrightarrow K_{*}^{\text {alg }}(\mathbb{Z})$ induced by the unital ring homomorphism $\widetilde{I} \longrightarrow \mathbb{Z}$. This construction is functorial for nonunital ring homomorphisms. For the definition of the relative $K$-groups $K_{n}^{\operatorname{alg}}(\Lambda, J)$, where $J$ is a two-sided ideal in the unital ring $\Lambda$, we refer, for $n \geq 0$, to [34. Defs. 1.5.3 and 5.2.14]; for $n>0$, one sets $K_{-n}^{\text {alg }}(\Lambda, J):=K_{-n}^{\text {alg }}(J)$ (hiding the fact that $K_{-n}^{\mathrm{alg}}$ satisfies excision), see [34, Def. 3.3.1]. The above split exact sequence induces a canonical isomorphism

$$
K_{*}^{\mathrm{alg}}(\widetilde{I}) \cong K_{*}^{\mathrm{alg}}(\widetilde{I}, I) \oplus K_{*}^{\mathrm{alg}}(\mathbb{Z}),
$$

as follows from the long exact sequence in algebraic $K$-theory, see [34, Thm. 3.3.4]. Since $K_{0}^{\mathrm{alg}}$ satisfies excision too (see [34. Thm. 1.5.9]), and since the ring $\mathbb{Z}$ is regular, so that its negative algebraic $K$-groups all vanish (see [34, Ex. 3.1.2 (4) and Def. 3.3.1]), we get

$$
K_{-n}^{\mathrm{alg}}(\widetilde{I}) \cong \begin{cases}K_{-n}^{\mathrm{alg}}(I), & \text { if } n>0 \\ K_{0}^{\mathrm{alg}}(I) \oplus \mathbb{Z}, & \text { if } n=0 .\end{cases}
$$

Recall that the cone of $\mathbb{Z}$ is the unital ring $C(\mathbb{Z})$ consisting of the infinite matrices $\left(a_{i j}\right)_{i, j \in \mathbb{N}}$ with only finitely many non-zero (integer-valued) entries in each row and in each column. The suspension of $\mathbb{Z}$ is the quotient $S(\mathbb{Z}):=C(\mathbb{Z}) / \mathbb{M}(\mathbb{Z})$, where $\mathbb{M}(\mathbb{Z})$ is the two-sided ideal of finite matrices, i.e. the union $\bigcup_{n>1} \mathbb{M}_{n}(\mathbb{Z})$ in $C(\mathbb{Z})$. The main feature of $C(\mathbb{Z})$ is that it is unital with vanishing algebraic $K$-theory (including in negative degree). For $k \geq 1$, the $k$-fold suspension of a unital ring $\Lambda$ is the unital ring $S^{k}(\Lambda):=\Lambda \otimes_{\mathbb{Z}} S(\mathbb{Z})^{\otimes_{\mathbb{Z}} k}$ (with the obvious ring structure). The $\operatorname{ring} S^{k}(\Lambda)$ satisfies the following property:

$$
K_{n}^{\mathrm{alg}}\left(S^{k}(\Lambda)\right) \cong K_{n-k}^{\mathrm{alg}}(\Lambda) \quad(n \in \mathbb{Z}) .
$$


We also need the fact that algebraic $K$-theory is additive in the sense that there is a natural isomorphism $K_{*}^{\text {alg }}\left(\Lambda_{1} \times \Lambda_{2}\right) \cong K_{*}^{\text {alg }}\left(\Lambda_{1}\right) \oplus K_{*}^{\text {alg }}\left(\Lambda_{2}\right)$, for any two unital rings $\Lambda_{1}$ and $\Lambda_{2}$. This property is clear in degree zero and then follows from the definition in negative degrees; for positive degrees, see [25. Prop. 1.2.3]. One further has a canonical decomposition $\mathrm{GL}\left(\Lambda_{1} \times \Lambda_{2}\right) \cong \mathrm{GL}\left(\Lambda_{1}\right) \times \mathrm{GL}\left(\Lambda_{2}\right)$, and similarly for $\mathrm{E}(-)$ and $\mathrm{St}(-)$, see [26] p. 326 and Prop. 12.8].

We have to discuss $C^{*}$-algebras in connection with algebraic $K$-theory. A $C^{*}$ algebra $\mathcal{A}$ is called stable if it is $*$-isomorphic to $\mathcal{A} \widehat{\otimes} \mathcal{K}$. Since $\mathcal{K} \cong \mathcal{K} \widehat{\otimes} \mathcal{K}$, for any $C^{*}$ algebra $\mathcal{A}$, the $C^{*}$-algebra $\mathcal{A} \widehat{\otimes} \mathcal{K}$ is stable. We now recall a deep result, namely the Karoubi Conjecture (proved in Suslin-Wodzicki [36, 37] - see Remark 3.1 below) :

The canonical "change-of- $K$-theory map" $K_{*}^{\text {alg }}(\mathcal{A}) \longrightarrow K_{*}^{\text {top }}(\mathcal{A})$ is an isomorphism, for any stable $C^{*}$-algebra $\mathcal{A}$.

(Note that this includes the negative $K$-groups $K_{-n}^{\text {alg }}$ and $K_{-n}^{\text {top }}$ with $n>0$.)

Remark 3.1. For the proof of our main results, we will not need the full power of the Karoubi Conjecture that $K_{n}^{\operatorname{alg}}(\mathcal{A} \widehat{\otimes} \mathcal{K}) \cong K_{n}^{\text {top }}(\mathcal{A} \widehat{\otimes} \mathcal{K})$ for any $C^{*}$-algebra $\mathcal{A}$ and any $n \in \mathbb{Z}$ - which has been proved, as we have just mentioned. Indeed, in the proofs, we will consider a certain stable $C^{*}$-algebra $\mathcal{F}$ and will only need the values of its algebraic $K$-theory for $n \leq 0$, because of the occurrence of an iterated suspension (see below). In 1979, Karoubi himself proved in [22] that his conjecture is true for $n \leq 0$ - in fact, this motivated the conjecture. Later, this was shown for $n=1$ in de la Harpe-Skandalis [15, and for $n=2$ in Karoubi 23 and also in Higson 18. In the latter it is also proved that the Karoubi Conjecture holds for Karoubi-Villamajor's algebraic $K$-theory. Finally, it was Suslin and Wodzicki who established the conjecture for Quillen's algebraic $K$-theory, in [36, 37. For related results, the reader may consult [20, 21, 40].

\section{Construction of the group $S$ of Theorem 1.1}

Here we provide the construction of the group $S$ occurring in Theorem 1.1 and in its complement, namely Theorem 6.1 below. This will justify the long recollections of Sections 2 and 3 since then, almost all properties arising in those statements will become "automatic", by the very construction.

To begin with, for an abelian group $M$, consider the nonunital $C^{*}$-algebra

$$
\mathcal{F}_{M}:=\mathcal{E}_{M} \widehat{\otimes} \mathcal{K},
$$

where $\mathcal{E}_{M}$ is as in Proposition 2.1 By the results quoted in Section 2 we have, for every $n \in \mathbb{Z}$,

$$
K_{2 n}^{\mathrm{alg}}\left(\mathcal{F}_{M}\right) \cong K_{2 n}^{\mathrm{top}}\left(\mathcal{E}_{M}\right) \cong M \quad \text { and } \quad K_{2 n+1}^{\mathrm{alg}}\left(\mathcal{F}_{M}\right) \cong K_{2 n+1}^{\mathrm{top}}\left(\mathcal{E}_{M}\right)=0 .
$$

Now let $A, B$ and $C$ be three abelian groups, prescribed as in Theorem 1.1. We require unital rings having the appropriate algebraic $K$-theory in low dimensions. For this purpose, we let

$$
R_{A}:=S^{4}\left(\widetilde{\mathcal{F}_{A}}\right), \quad R_{B}:=S^{4}\left(\widetilde{\mathcal{F}_{B}}\right) \quad \text { and } \quad R^{C}:=S^{5}\left(\widetilde{\mathcal{F}_{C}}\right)
$$

be the 4-fold (resp. 5-fold) algebraic suspension of the minimal unitalization of the nonunital rings $\mathcal{F}_{A}$ and $\mathcal{F}_{B}$ (resp. $\mathcal{F}^{C}$ ), see Section 3 . Assembling most of the results 
recalled in Sections 2] and 3] we obtain, for $n \leq 3$,

$$
K_{n}^{\mathrm{alg}}\left(R_{A}\right) \cong K_{n}^{\mathrm{top}}\left(\mathcal{E}_{A}\right) \cong\left\{\begin{array}{l}
A, \text { if } n \leq 2 \text { is even } \\
0, \text { if } n \leq 3 \text { is odd }
\end{array}\right.
$$

and similarly for $B$, while, for $n \leq 4$,

$$
K_{n}^{\mathrm{alg}}\left(R^{C}\right) \cong K_{n-1}^{\mathrm{top}}\left(\mathcal{E}_{C}\right) \cong \begin{cases}0, & \text { if } n \leq 4 \text { is even } \\ C, & \text { if } n \leq 3 \text { is odd } .\end{cases}
$$

We also note that for each $n \in \mathbb{Z}$, the abelian groups $K_{n}^{\mathrm{alg}}\left(R_{A}\right)$ and $K_{n}^{\mathrm{alg}}\left(R_{B}\right)$ (resp. $K_{n}^{\text {alg }}\left(R^{C}\right)$ ) contain a direct summand isomorphic to $K_{n-4}^{\text {alg }}(\mathbb{Z})\left(\right.$ resp. $K_{n-5}^{\text {alg }}(\mathbb{Z})$ ). Finally, for the group $S$ that we have to construct, we take

$$
S:=\operatorname{St}\left(R_{A}\right) \times \mathrm{E}\left(R_{B}\right) \times \operatorname{St}\left(R^{C}\right) .
$$

Note that $K_{n}^{\mathrm{alg}}\left(R^{C}\right)$ being zero, we have $S \cong \mathrm{St}\left(R_{A}\right) \times \mathrm{E}\left(R_{B}\right) \times \mathrm{E}\left(R^{C}\right)$.

\section{Proof of Theorem 1.1}

Before the proof, we introduce the following convenient terminology. We call a group $G n$-perfect for some $n \geq 1$ if its reduced integral homology vanishes in dimension $\leq n$, i.e. $\widetilde{H}_{j}(G)=0$ for all $j \leq n$; of course, 1-perfect is the same as perfect in the usual sense, while 2-perfect is often called superperfect. In the literature, the terms $n$-connected and $n$-acyclic are also to be found.

We may now prove, in turn, the statements (i)-(v) of Theorem 1.1

(i). The proof of Lemma 11 presented in [10 actually establishes the following slightly stronger statement than that asserted there.

Lemma 5.1. Let $H$ be a simple group that, for each $n \geq 2$, has a 2-perfect subgroup $L_{n}$ possessing an element of order $n$. Suppose that $G$ is a group containing $H$ in such a way that the normal closure of $H$ in $G$ is $G$ itself. Then every perfect central extension of $G$ is strongly torsion generated.

As in [10], this result may be applied to the case where $G=\mathrm{E}(\Lambda)$ for any unital ring $\Lambda$ (with $H \cong A_{\infty}$, see loc. cit.), to yield that every perfect central extension of $\mathrm{E}(\Lambda)$ is strongly torsion generated. For the present circumstance, we take $\Lambda=R_{A} \times R_{B} \times R^{C}$, and deduce that the perfect central extension $S$ is strongly torsion generated.

(ii). Since for any unital ring $\Lambda$ the group $\mathrm{E}(\Lambda)$ has trivial centre, while the centre of $\operatorname{St}(\Lambda)$ is precisely $K_{2}^{\mathrm{alg}}(\Lambda)$, we immediately have $A$ as the centre of $S$.

(iii). As quoted in Section 2 the groups $\mathrm{E}(\Lambda)$ and $\operatorname{St}(\Lambda)$ are perfect, for any unital ring $\Lambda$; and a finite product of perfect groups is perfect. (Here, one can also recall from [10, Lem. 7] that every strongly torsion generated group is perfect.)

(iv) \& (v). Lastly, for the claims about the homology groups of $S$, we observe from Hurewicz isomorphisms that the first nonzero reduced homology groups of $\operatorname{St}\left(R_{A}\right)$, of $\mathrm{E}\left(R_{B}\right)$ and of $\operatorname{St}\left(R^{C}\right)$ occur in dimensions 4,2 and 3 respectively. Moreover, combining with the epimorphism in the next dimension, we have

$$
\begin{aligned}
& -H_{2}\left(\mathrm{E}\left(R_{B}\right)\right) \cong K_{2}^{\mathrm{alg}}\left(R_{B}\right) \cong B \quad \text { and } \quad H_{3}\left(\mathrm{E}\left(R_{B}\right)\right)=0 \\
& \text { - } H_{3}\left(\operatorname{St}\left(R^{C}\right)\right) \cong K_{3}^{\mathrm{alg}}\left(R^{C}\right) \cong C \quad \text { and } \quad H_{4}\left(\operatorname{St}\left(R^{C}\right)\right)=0 .
\end{aligned}
$$


Hence, the desired results are immediate from the Künneth Theorem. This completes the proof.

Remark 5.2. (i) Observe that for $n_{A} \geq 2$ as large as we like, we can replace $S^{4}\left(\widetilde{\mathcal{F}_{A}}\right)$ by $S^{2 n_{A}}\left(\widetilde{\mathcal{F}_{A}}\right)$. Note also that for $n_{A} \geq 3$,

$$
H_{4}\left(\operatorname{St}\left(R_{A}\right)\right) \cong K_{4}^{\mathrm{alg}}\left(R_{A}\right) \cong A
$$

(however, for $n_{A}=2$ we get $A \oplus \mathbb{Z}$ ). We can play the same game with $B$; while for $C$ we can take $S^{2 n_{C}+1}\left(\widetilde{\mathcal{F}_{C}}\right)$ with $n_{C} \geq 2$. We need $n_{A}, n_{B} \geq 3$ in Theorem 6.1(i) below.

(ii) For $n \geq 2 n_{A}, K_{n}^{\text {alg }}\left(R_{A}\right)$ contains a direct summand $K_{n-2 n_{A}}^{\text {alg }}(\mathbb{Z})$, and so, when $n-2 n_{A} \equiv 1(\bmod 4)$ and $n-2 n_{A} \geq 5$, a direct summand isomorphic to $\mathbb{Z}$, see [34] Thms 5.3.12 and 5.3.13]; similarly with $B$ and $C$.

(iii) When $A=0$, the unital ring $R_{A}$ is isomorphic to the $2 n_{A}$-fold suspension of the ring of integers, $S^{2 n_{A}}(\mathbb{Z})$; and similarly for $R_{B}$ (resp. $R^{C}$ ) when $B=0$ (resp. $C=0$ ).

(iv) There are two drawbacks to our construction of the group $S$ above: first, our construction of $S$ is not functorial in $A, B$ and $C$ (see however Remark 2.3); secondly, for $A, B$ and $C$ countable (and even finite), the group $S$ is not countable.

\section{Further Consequences of the COnstruction}

We now show that the group $S$ of Theorem 1.1 can be constructed in such a way that further properties hold, that are stated as Theorem 6.1 below.

In this section, assuming that we have taken $n_{A}, n_{B} \geq 3$ in the notation of Remark [5.2(i), we prove the next result, which complements Theorem 1.1

Theorem 6.1. The group $S$ of Theorem 1.1 has the following further properties:

(i) when $B=0$, one has $H_{4}(S) \cong A$;

(ii) for infinitely many dimensions $n$, the homology group $H_{n}(S)$ contains an infinite cyclic direct summand;

(iii) every finite-dimensional complex representation of $S$ is trivial;

(iv) $S / A$ is strongly torsion generated, centreless, and has $H_{2}(S / A) \cong A \times B$.

Proof. As in Remark [5.2(i), we choose integers $n_{A}, n_{B} \geq 3$ and $n_{C} \geq 2$, and take

$$
R_{A}=S^{2 n_{A}}\left(\widetilde{\mathcal{F}_{A}}\right), \quad R_{B}=S^{2 n_{B}}\left(\widetilde{\mathcal{F}_{B}}\right) \quad \text { and } \quad R^{C}=S^{2 n_{C}+1}\left(\widetilde{\mathcal{F}_{C}}\right) .
$$

(i). Since $B=0$, by the Hurewicz isomorphism, the first nonzero reduced homology group of $\mathrm{E}\left(R_{B}\right)$ is in dimension $2 n_{B} \geq 6$, so that $H_{4}\left(\mathrm{E}\left(R_{B}\right)\right)=0$. (Of course, one could also modify $S$ by omitting all usage of $R_{B}$ in this case.) As a consequence and since $n_{A} \geq 3$, the isomorphism $H_{4}\left(\operatorname{St}\left(R_{A}\right)\right) \cong A$ of Remark 5.2(i) combines with the Künneth Theorem to give the result.

(ii). By [1] Thm. 2.1], for $n \geq 3$, there are homomorphisms

$$
K_{n}^{\mathrm{alg}}(\Lambda) \longrightarrow H_{n}(\mathrm{E}(\Lambda)) \longrightarrow K_{n}^{\mathrm{alg}}(\Lambda) \text { and } K_{n}^{\mathrm{alg}}(\Lambda) \longrightarrow H_{n}(\operatorname{St}(\Lambda)) \longrightarrow K_{n}^{\mathrm{alg}}(\Lambda) \text {, }
$$

such that in each case the composite is multiplication by a positive integer. This means that elements of infinite order in $K_{n}^{\text {alg }}(\Lambda)$ are mapped to elements of infinite order by the composite, and so must have images of infinite order in $H_{n}(\mathrm{E}(\Lambda)$ ) and in $H_{n}(\operatorname{St}(\Lambda))$, respectively. Hence, by Remark 5.2(ii), no matter which of the 
possible values for $n_{A}, n_{B}$ and $n_{C}$ we choose in our construction, this yields an infinite cyclic direct summand (and possibly three such summands) in $H_{n}(\mathrm{E}(\Lambda)$ ), in $H_{n}(\mathrm{St}(\Lambda))$, and therefore in $H_{n}(S)$, for infinitely many values of $n$.

(iii). Because a surjective unital ring homomorphism, such as that from the cone of a ring to its suspension, induces a surjection of Steinberg groups, it follows from the construction that the group $S$ is a homomorphic image of the Steinberg group

$$
\operatorname{St}\left(C\left(S^{2 n_{A}-1}\left(\widetilde{\mathcal{F}_{A}}\right) \times S^{2 n_{B}-1}\left(\widetilde{\mathcal{F}_{B}}\right) \times S^{2 n_{C}}\left(\widetilde{\mathcal{F}_{C}}\right)\right)\right) \text {. }
$$

This group is acyclic and torsion-generated; therefore, by the main result of [6], it has no nontrivial finite-dimensional complex representation (cf. [7, p. 191]).

(iv). We have $\operatorname{St}\left(R_{A}\right) / A \cong \mathrm{E}\left(R_{A}\right)$ whence it follows that the quotient $S / A$ is isomorphic to $\mathrm{E}\left(R_{A} \times R_{B} \times R^{C}\right)$ and is strongly torsion generated, centreless, with second homology group isomorphic to $K_{2}^{\text {alg }}\left(R_{A} \times R_{B} \times R^{C}\right) \cong A \times B$.

The interest of item (ii) is heightened by the following observation. In [10, to construct a strongly torsion generated group $S$ with prescribed centre $A$, one starts with a centreless strongly generated group $S^{\prime}$ with its reduced integral homology concentrated in dimension 2 and isomorphic to $A$, and then one takes for $S$ the universal central extension of $S^{\prime}$ (see details in 10, Proof of Cor. 16]). In that sense, if $A$ has very few nonvanishing (resp. nontorsion) integral homology groups (for example, if $A$ is free abelian of finite rank), then by the Lyndon-HochschildSerre spectral sequence one can expect $S$ to have very few nonvanishing (resp. nontorsion) integral homology groups as well. The argument of 10 obliges one to be in this situation in order to establish that $S$ is strongly torsion generated. In contrast, for the present construction of $S$, infinite higher homology groups are inescapable.

\section{SOME OPEN QUESTIONS}

The following questions are prompted by Theorem 1.1 For each of them, the further requirement of a functorial construction is also of interest.

Question 7.1. Can one find a countable group $S$ as in the statement of Theorem 1.1 for $A, B$ and $C$ finite or countable? What about if we replace, everywhere, the word "countable" by "finitely generated", or "finitely presented"?

As a matter of comparison, it is known that any group (resp. countable group, finitely generated group, finitely presented group, geometrically finite group) embeds in an acyclic group (resp. countable acyclic group, seven-generator acyclic group, finitely presented acyclic group, geometrically finite acyclic group), see [2] . (A group $G$ is called geometrically finite if there exists a model for its classifying space that is a finite $C W$-complex; in particular, $G$ is then torsion-free.) Since a strongly torsion generated group, by its very definition, contains "a lot of torsion", Question [7.1] for "geometrically finite" has a negative answer.

Question 7.2. Given $n \geq 3$, is it possible to define an $n$-perfect strongly torsion generated group $S$ with centre and $H_{n+1}(S)$ prescribed abelian groups?

The cases $n=1$ and $n=2$ have been achieved above. In the case $n=2$, the extension $A \longmapsto S \rightarrow S / A$ is the universal central extension of the perfect group $S / A$, and so, $H_{2}(S / A) \cong A$.

The case $n=\infty$ (so to speak) of the above question merits special attention. 
Question 7.3. Given an abelian group $A$, is it possible to construct an acyclic strongly torsion generated group $S$ with centre isomorphic to $A$ ?

Again, we can compare this with the fact, proved in [5], that any abelian group is the centre of some acyclic group, in a functorial and explicit way.

Question 7.4. Given an abelian group $M$ and $n \geq 3$, does there exist a group $G$ with $G /[G, G] \cong M$, and such that $[G, G]$ is $n$-perfect strongly torsion generated with trivial centre? Can one further require $[G, G]$ to be acyclic?

Again, we have already dealt with the case $n=2$, where, as group $G$, we take the infinite general linear group GL $\left(S^{3}\left(\widetilde{\mathcal{F}_{M}}\right)\right)$, which satisfies

$$
G /[G, G]=K_{1}^{\mathrm{alg}}\left(S^{3}\left(\widetilde{\mathcal{F}_{M}}\right)\right) \cong M \quad \text { and } \quad[G, G]=\mathrm{E}\left(S^{3}\left(\widetilde{\mathcal{F}_{M}}\right)\right)=\operatorname{St}\left(S^{3}\left(\widetilde{\mathcal{F}_{M}}\right)\right) .
$$

Finally, here are two questions that we plan to address in subsequent work.

Question 7.5. Is it possible similarly to construct a strongly torsion generated group $S$ with given centre and having more prescribed homology than in Theorem 1.1]?

Question 7.6. What kind of information do the ring $\widetilde{\mathcal{F}_{M}}$ and its $K$-theory convey about the abelian group $M$ ?

\section{REFERENCES}

[1] D. Arlettaz. The Hurewicz homomorphism in algebraic K-theory, J. Pure Appl. Algebra $\mathbf{7 1}$ (1991), 1-12.

[2] G. Baumslag, E. Dyer and A. Heller. The topology of discrete groups, J. Pure Appl. Algebra 16 (1980), 1-47.

[3] G. Baumslag, E. Dyer and C.F. Miller III. On the integral homology of finitely presented groups, Topology 22 (1983), 27-46.

[4] A. J. Berrick. An approach to algebraic K-theory. Pitman Research Notes Math. 56 (London, 1982).

[5] A. J. Berrick. Two functors from abelian groups to perfect groups, J. Pure Applied Algebra 44(1987), 35-43.

[6] A. J. Berrick. Remarks on the structure of acyclic groups, Bull. London Math. Soc. 22 (1990), $227-232$.

[7] A. J. Berrick. Torsion generators for all abelian groups, J. Algebra 139 (1991), 190-194.

[8] A. J. Berrick. A topologist's view of perfect and acyclic groups, In Invitations to Geometry and Topology, ed. M. R. Bridson and S. M. Salamon, Oxford Graduate Texts in Math. 5, Oxford Univ. Press (Oxford, 2002), chapter 1, 1-28.

[9] A. J. Berrick and P. H. Kropholler. Groups with infinite homology, in Cohomological Methods in Homotopy Theory (BCAT98, Bellaterra 1998), Progress in Math. 196, Birkhauser (Basel, 2001), 27-33.

[10] A. J. Berrick and C. F. Miller III. Strongly torsion generated groups, Math. Proc. Cambridge Philos. Soc. 111 (1992), no. 2, 219-229.

[11] L. Claborn. Every abelian group is a class group, Pacific J. Math. 118 (1966), 219-222.

[12] S. Eilenberg and S. Mac Lane. On the groups $H(\Pi, n)$. II. Methods of computation, Ann. of Math. 60 (1954), 49-139.

[13] L. Evens. The cohomology ring of a finite group, Trans. Amer. Math. Soc. 101 (1961), 224-239.

[14] S. M. Gersten. $K_{3}$ of a ring is $H_{3}$ of the Steinberg group, Proc. Am. Math. Soc. 37 (1973), 366-368.

[15] P. de la Harpe and G. Skandalis. Sur la simplicité essentielle du groupe des inversibles et du groupe unitaire dans une $C^{*}$-algèbre simple, J. Func. Anal. 62 (1985), 354-378.

[16] C. H. Giffen. Contributed problems. In Proc. Northwestern Univ. Homotopy Theory Conf., Contemp. Math. 19, Amer. Math. Soc. (RI, 1982), 447. 
[17] K. K. Hickin. Universal locally finite central extensions of groups, Proc. London Math. Soc. (3) 52 (1986), 53-72.

[18] N. Higson. Algebraic $K$-theory of stable $C^{*}$-algebras, Adv. in Math. 67 (1988), 1-140.

[19] P. J. Hilton and U. Stammbach. A Course in Homological Algebra, 2nd ed. Graduate Texts in Math. 4, Springer (New York, 1997).

[20] H. Inassaridze. Algebraic K-theory of normed algebras, K-Theory 21 (2000), 25-56.

[21] H. Inassaridze and T. Kandelaki. $K$-theory of stable generalized operator algebras, $K$-Theory 27 (2002), 103-110.

[22] M. Karoubi. $K$-théorie algébrique de certaines algèbres d'opérateurs, Lecture Notes in Math. 725, Springer (Berlin, 1979), 254-290.

[23] M. Karoubi. Homologie des groupes discrets associés à des algèbres d'opérateurs, J. Operator Theory 15 (1986), 109-161.

[24] C. R. Leedham-Green. The class group of Dedekind domains, Trans. Amer. Math. Soc. 163 (1972), 493-500.

[25] J.-L. Loday. K-théorie algébrique et représentations de groupes, Ann. Sc. Ec. Norm. Sup. 9 (1976), 309-377.

[26] B. A. Magurn. An algebraic introduction to K-theory. Encyclopedia of Math. and its Applications 87, Cambridge Univ. Press, (Cambridge, 2002).

[27] G. Malle and B. H. Matzat. Inverse Galois Theory. Springer Monographs in Math., Springer (Berlin, 1999).

[28] R. J. Milgram. The cohomology of the Mathieu group $M_{23}$, J. Group Theory 3 (2000), 7-26.

[29] H. Miller. The Sullivan conjecture on maps from classifying spaces. Ann. of Math. (2) 120 (1984), 39-87.

[30] J. Milnor. Introduction to algebraic K-theory. Annals of Mathematics Studies 72, Princeton Univ. Press, Princeton, 1971.

[31] B. H. Neumann. A note on algebraically closed groups, J. London Math. Soc. 27 (1952), 247-249.

[32] R. E. Phillips. Existentially closed locally finite central extensions, multipliers and local systems, Math. Z. 187 (1984), 383-392.

[33] M. Rørdam, F. Larsen and N. Laustsen. An introduction to $K$-theory for $C^{*}$-algebras. London Math. Soc. Student Texts 49, Cambridge Univ. Press (Cambridge, 2000).

[34] J. Rosenberg. Algebraic K-theory and its applications. Graduate Texts in Math. 147, Springer (Berlin, 1994).

[35] W. R. Scott. Algebraically closed groups, Proc. Amer. Math. Soc. 2 (1951), 118-121.

[36] A. A. Suslin and M. Wodzicki. Excision in algebraic $K$-theory and Karoubi's conjecture, Proc. Nat. Acad. Sci. U.S.A. 87 (1990), no. 24, 9582-9584.

[37] A. A. Suslin and M. Wodzicki. Excision in algebraic K-theory, Ann. of Math. (2) 136 (1992), no. $1,51-122$.

[38] R. G. Swan. The nontriviality of the restriction map in the cohomology of groups, Proc. Amer. Math. Soc. 11 (1960), 885-887.

[39] R. M. Switzer. Algebraic topology - homotopy and homology. Die Grundlehren der math. Wissenschaften, Band 212, Springer (Berlin, 1975).

[40] J. Tapia. $K$-théorie algébrique négative et $K$-théorie topologique de l'algèbre de Fréchet des opérateurs régularisants, Ann. Sci. École Norm. Sup. 30 (1997), 241-277.

[41] N. E. Wegge-Olsen. $K$-theory and $C^{*}$-algebras. A friendly approach. Oxford Science Publications, Oxford Univ. Press (Oxford, 1993).

Department of Mathematics, National University of Singapore, Singapore 117543, Republic of Singapore

E-mail address: berrick@math.nus.edu.sg

University of Lausanne, IGAT (Institute for Geometry, Algebra and Topology), BCH, EPFL, CH-1015 Lausanne, Switzerland

E-mail address: michel.matthey@ima.unil.ch 